\title{
Pediatric Transthoracic Cardiac Vector Flow Imaging - A Preliminary Pictorial Study
}

\section{(c) (1) (ㅇ) $\ominus$}

Authors

Kristoffer Lindskov Hansen ${ }^{1}$, Klaus Juul2 ${ }^{2}$, Hasse Møller-Sørensen ${ }^{3}$, Jens C. Nilsson ${ }^{3}$, Jørgen Arendt Jensen ${ }^{4}$, Michael Bachmann Nielsen ${ }^{1}$

Affiliations

1 Department of Diagnostic Radiology, Copenhagen University Hospital, Copenhagen, Denmark

2 Department of Pediatric Cardiology, Copenhagen University Hospital, Copenhagen, Denmark

3 Department of Cardiothoracic Anesthesiology, Copenhagen University Hospital, Copenhagen, Denmark

4 Center for Fast Ultrasound Imaging, Technical University of Denmark, DTU Elektro, Lyngby, Denmark

Key words

Vector Flow Imaging, Transverse Oscillation, Transthoracic echocardiography, Cardiac flow, Congenital heart disease

received 12.03 .2018

revised 15.05 .2018

accepted 01.07.2018

Bibliography

DOI https://doi.org/10.1055/a-0656-5430

Ultrasound Int Open 2019; 5: E20-E26

(c) Georg Thieme Verlag KG Stuttgart · New York

ISSN 2199-7152

Correspondence

Dr. Kristoffer Lindskov Hansen, MD

Department of Diagnostic Radiology
Copenhagen University Hospital

Blegdamsvej 9

Copenhagen, DK-2100

Denmark

Tel.: +45/354/53 545

lindskov@gmail.com

\section{ABSTRACT}

Purpose Conventional pediatric echocardiography is crucial for diagnosing congenital heart disease (CHD), but the technique is impaired by angle dependency. Vector flow imaging (VFI) is an angle-independent noninvasive ultrasound alternative for blood flow assessment and can assess complex flow patterns not visible on conventional Doppler ultrasound.

Materials and Methods 12 healthy newborns and 3 infants with CHD were examined with transthoracic cardiac VFI using a conventional ultrasound scanner and a linear array.

Results VFI examinations revealed common cardiac flow patterns among the healthy newborns, and flow changes among the infants with CHD not previously reported with conventional echocardiography.

Conclusion For assessment of cardiac flow in the normal and diseased pediatric heart, VFI may provide additional information compared to conventional echocardiography and become a useful diagnostic tool.

\section{Introduction}

Congenital heart disease (CHD) is the most common congenital defect in newborns with a prevalence of 8 out of 1000 live births [1]. The fundamental diagnostic tool in the management of children with CHD is echocardiography, performed during pregnancy, in newborns, and in infants [2,3]. Echocardiography is a noninvasive, cost-effective, available and highly sensitive cardiovascular tool based on B-mode imaging for anatomical evaluation, and Doppler ultrasound (US) for blood flow evaluation [4]. However, conventional Doppler US only estimates the flow component of blood motion along the direction of the emitted US beam and is consequently angle-dependent. Thus, assumptions of flow direction are necessary for flow quantification, and the visualization of complex flow patterns is limited [5].

Several US approaches for angle-independent velocity estimation have previously been proposed [5-7]. One US solution for angle-independent vector velocity estimation called transverse oscillation (TO) was introduced by Jensen and Munk [8]. The method implemented on a commercial scanner has previously been used in-vivo for intraoperative cardiac imaging in adults, but the method has not previously been employed in pediatric echocardiography [9-14].

In this study, healthy newborns and infants with CHD were examined with transthoracic US using VFI for qualitative cardiac flow evaluation. The study aim was to investigate if VFI can be used as a 
tool for pediatric cardiovascular assessment concerning the visualization of complex cardiac flow patterns in newborns and of cardiac flow changes in infants with CHD.

\section{Materials and Methods}

\section{Volunteers and patients}

This prospective study was performed after approval by the $\mathrm{Na}$ tional Committee on Biomedical Research Ethics (no. 16035388). 12 healthy full-term newborns ( 4 girls and 8 boys; age: $>37$ weeks of gestation) scanned within the first week after birth, and 3 infants with $\mathrm{CHD}$ were included with informed consent obtained from the parents. The infants with CHD were all boys and included: (I) one with a ventricular septal defect (VSD) (age: 4 months), (II) one with tetralogy of Fallot (ToF) (age: 1 month), (III) one with coarctation of the aorta (age: 2 months).

\section{Vector flow imaging}

The VFI method transverse oscillation, proposed by Jensen and Munk [8], is an angle-independent vector velocity method based on conventional Doppler pulse emission, which estimates both the axial and the transverse velocity component from each received echo. The axial velocity component is found as in conventional Doppler US, while the transverse velocity component is found by changing the sensitivity of the receiving transducer elements, and with the use of a special estimator [15]. Combining the axial and the transverse velocity components for each point within a region of interest provides a $2 \mathrm{D}$ vector velocity map of angle-independent blood velocities in the measurement plane.

A conventional US scanner (ProFocus 2202 UltraView, BK Medical, Herlev, Denmark) with a linear transducer (8670, BK Medical, Herlev, Denmark) was used for transthoracic cardiac VFI recordings and provided for this study free of charge by the manufacturer. Each examination included an apical four-chamber view, an apical five-chamber view including the left ventricular outflow tract (LVOT), a parasternal long-axis view of the right ventricular outflow tract (RVOT), parasternal long- and short-axis views of the aortic root and ascending aorta, and a suprasternal view of the aortic arch. For each scan, the color box, depth setting, gain, and pulse repetition frequency were adjusted for vector velocity estimation. For all measurements, the center frequency for B-mode imaging was
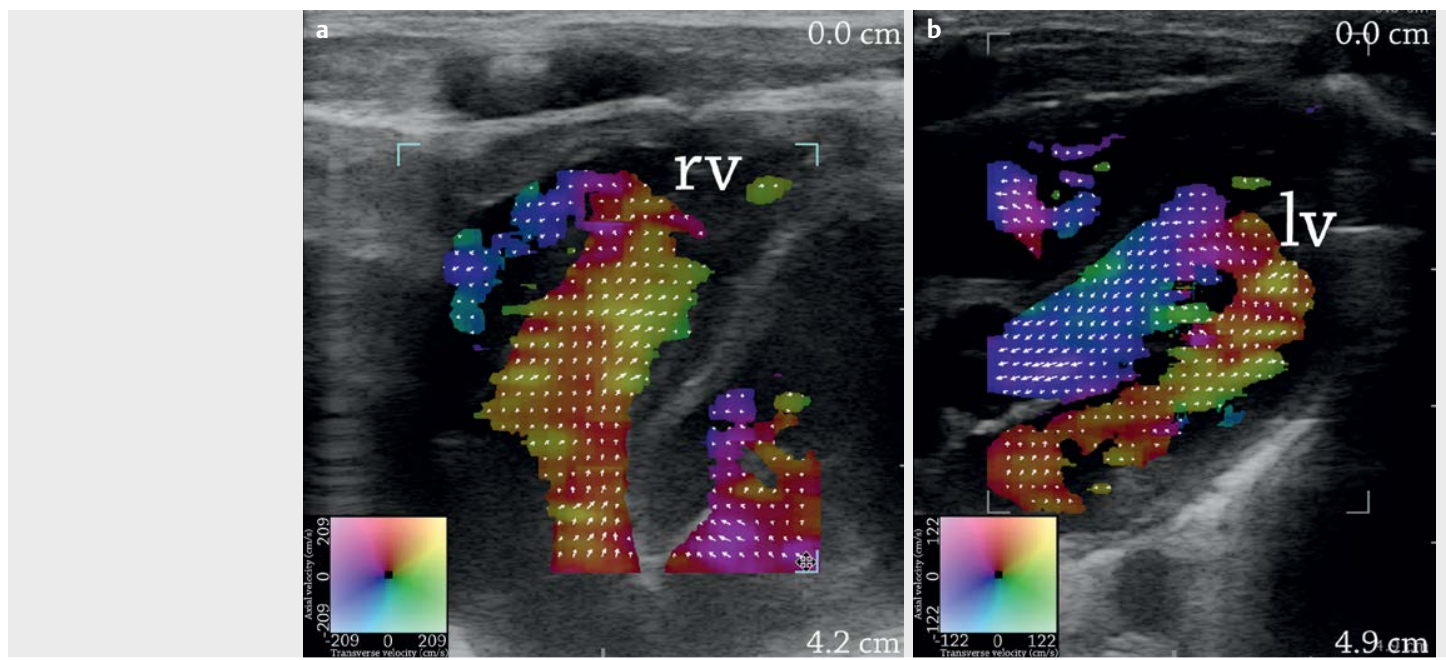

- Fig. 1 Blood flow in the right $\mathbf{a}$ and left $\mathbf{b}$ ventricle. Frames are recorded on two healthy newborns in apical four-chamber view. The direction and velocity of the blood flow are given by the corresponding color maps and indicated by the superimposed vector arrows. rv $=$ right ventricle, Iv=left ventricle.
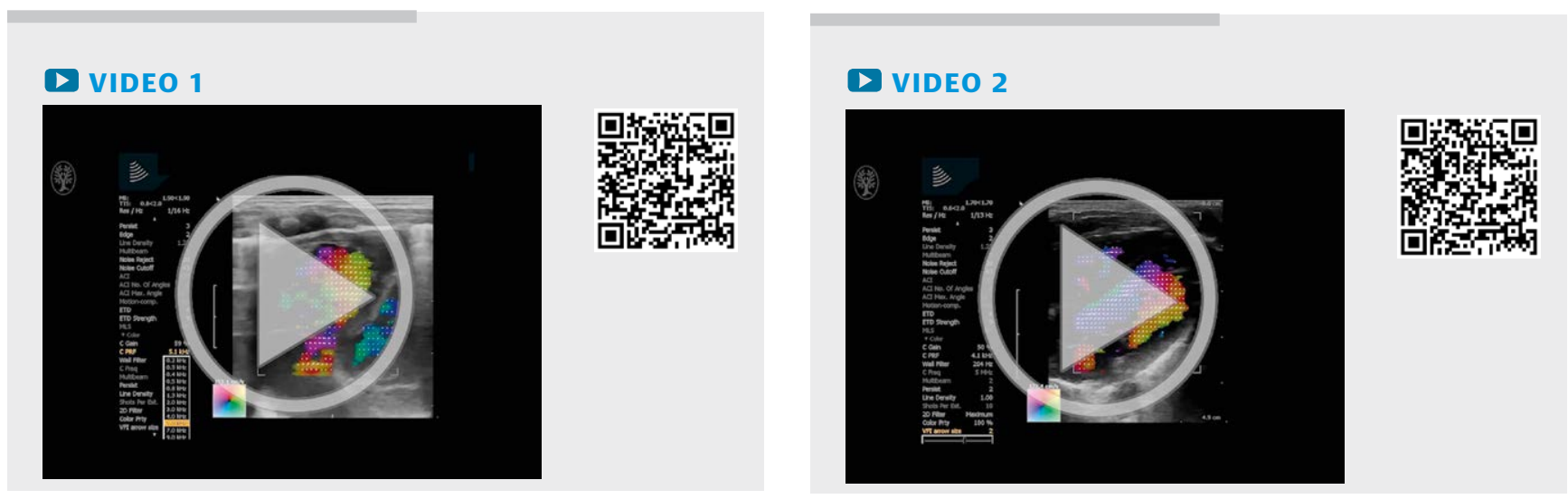
$9 \mathrm{MHz}$, and $5 \mathrm{MHz}$ for VFI. The temporal resolution of the VFI estimation was 16 frames/s, and the maximum scan depth was approximately $5 \mathrm{~cm}$ due to the available transducer setup. Each scan sequence of $14 \mathrm{~s}$ of recording corresponded to 225 frames. On the US scanner, vector velocity estimates were displayed in real time, but without any quantification of velocities available, and a visual analysis of the cardiac vector flow was performed off-line to outline common cardiac flow patterns in normal hearts and examples of flow changes in hearts with congenital disease. In this single-center preliminary observational study, the focus was on feasibility and description of the observed VFI findings in the studied pediatric patient group. In view of the small and heterogenic patient group, no statistical analysis was performed.

\section{Results}

Common flow patterns were seen for the healthy newborns as shown in $>$ Fig. 1a, b and $\triangleright$ Video. 1, 2. During the contraction of the left atrium, the flow exited the mitral valve to the left ventricle with a jet along the free wall to the apex. During the simultaneous contraction of the right atrium, the flow exited the tricuspid valve to the right ventricle with a jet along the posterior part of the septum to the apex ( $\vee$ Video. 1). During the ventricular contraction, the flow in the left ventricle returned along the septum to the LVOT ( $\triangleright$ Video. 2), while the flow in the right ventricle returned anteriorly along the free wall to the RVOT.

In both LVOT and RVOT, the flow was laminar, and as expected, small vortices were seen behind the valves in the pulmonary trunk $(\triangleright$ Video. $\mathbf{3})$ and the ascending aorta ( $\triangleright$ Fig. 2a, b). Six newborns were scanned with a suprasternal view over the aortic arch, where laminar flow was seen without systolic retrograde flow ( $>$ Fig. $2 c$ and $\triangleright$ Video. 4 ). In the short-axis view, helical flow was seen in the ascending aorta. The flow was clockwise for all except one newborn, who had a Dean-like flow with opposing vortices. Dean-like flow is a flow phenomenon of two counter-rotating vortices of the secondary flow described for curved vessels [16]. Four newborns were also scanned in the short-axis view over the left ventricle. The newborn with Dean-like flow in the ascending aorta had counter-clockwise rotations in the left ventricle, while two newborns had clockwise and one had Dean-like rotations ( $\mathbf{F i g . ~} \mathbf{3 a}, \mathbf{b}$ ).
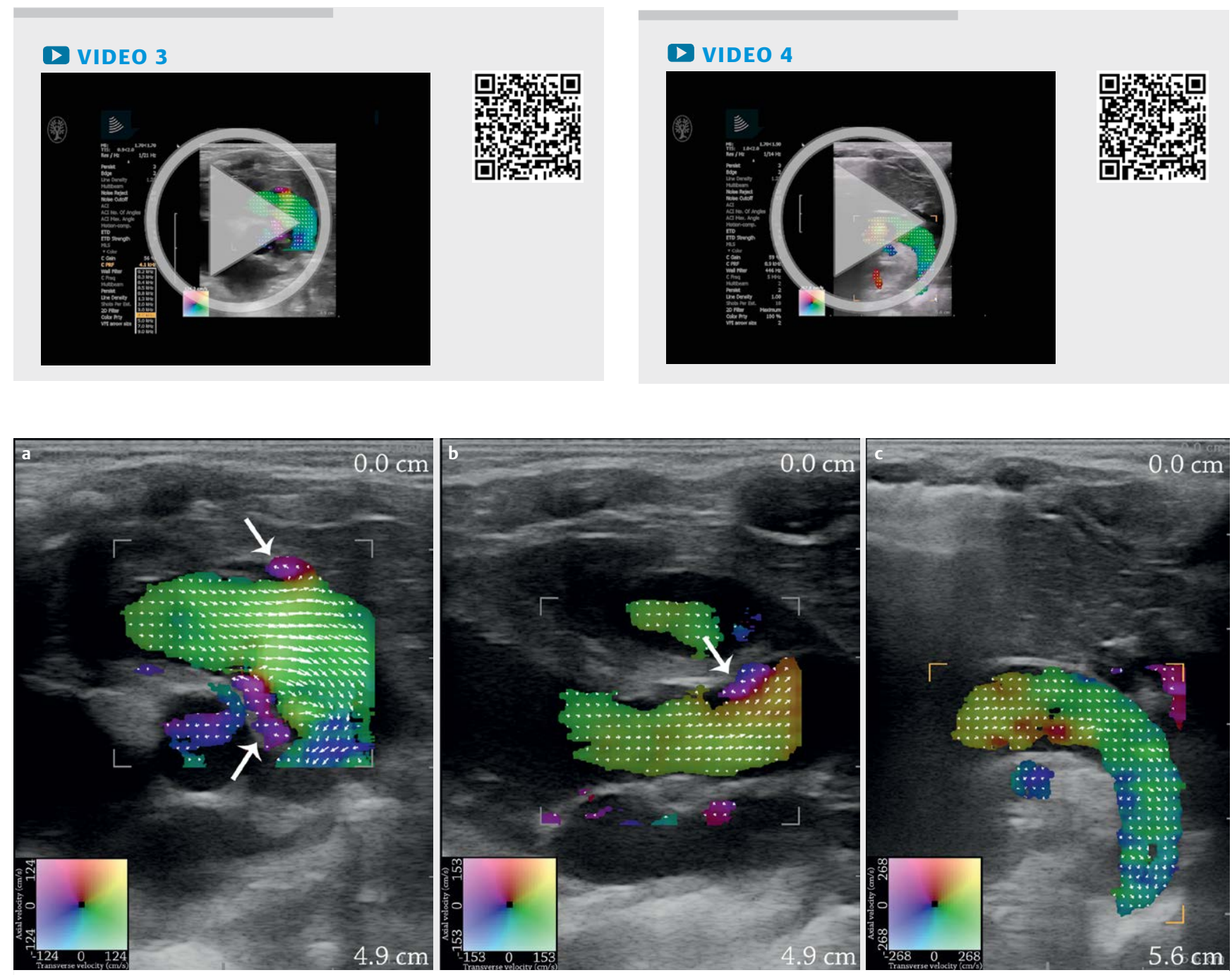

-Fig. $\mathbf{2}$ The flow in the pulmonary trunk $\mathbf{a}$, the ascending aorta $\mathbf{b}$, and aortic arch $\mathbf{c}$ of three healthy newborns. $\mathbf{a}$ and $\mathbf{b}$ are parasternal views, while $\mathbf{c}$ is a suprasternal view. Vortices behind the valves in the pulmonary trunk $\mathbf{a}$ and the ascending aorta $\mathbf{b}$ are marked with white arrows. 

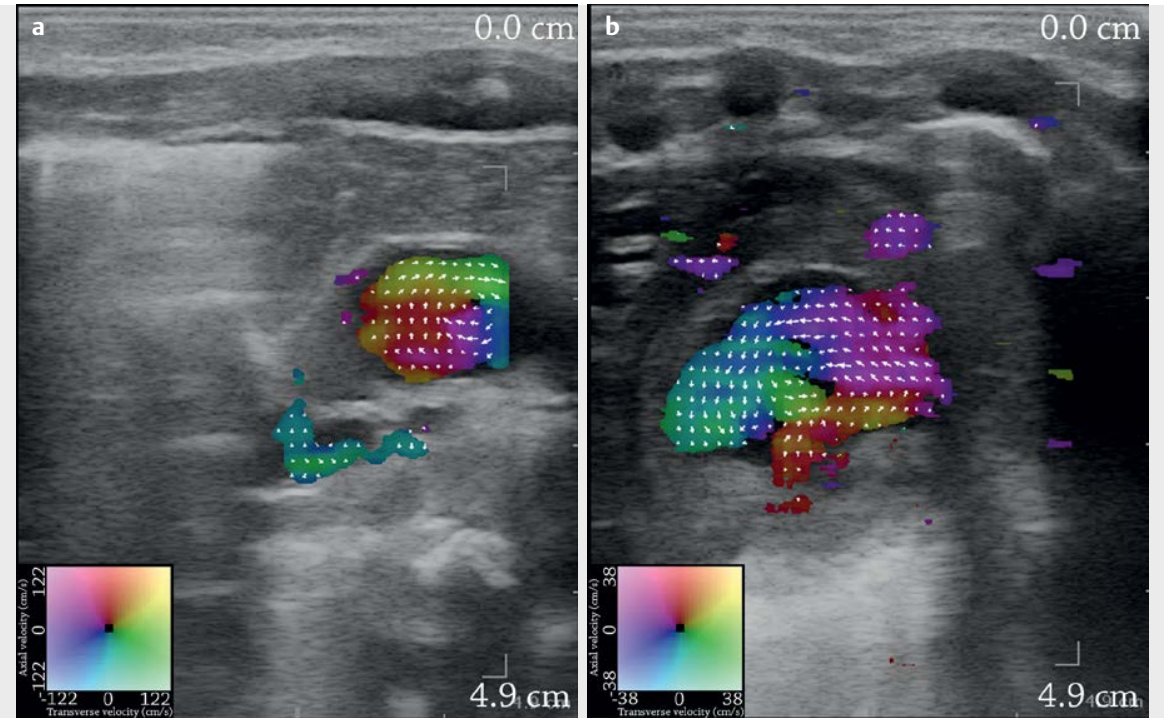

-Fig. 3 Clockwise rotational flow in the ascending aorta $\mathbf{a}$, and slow counter-clockwise rotational flow in the left ventricle b in short-axis parasternal views of two healthy newborns.
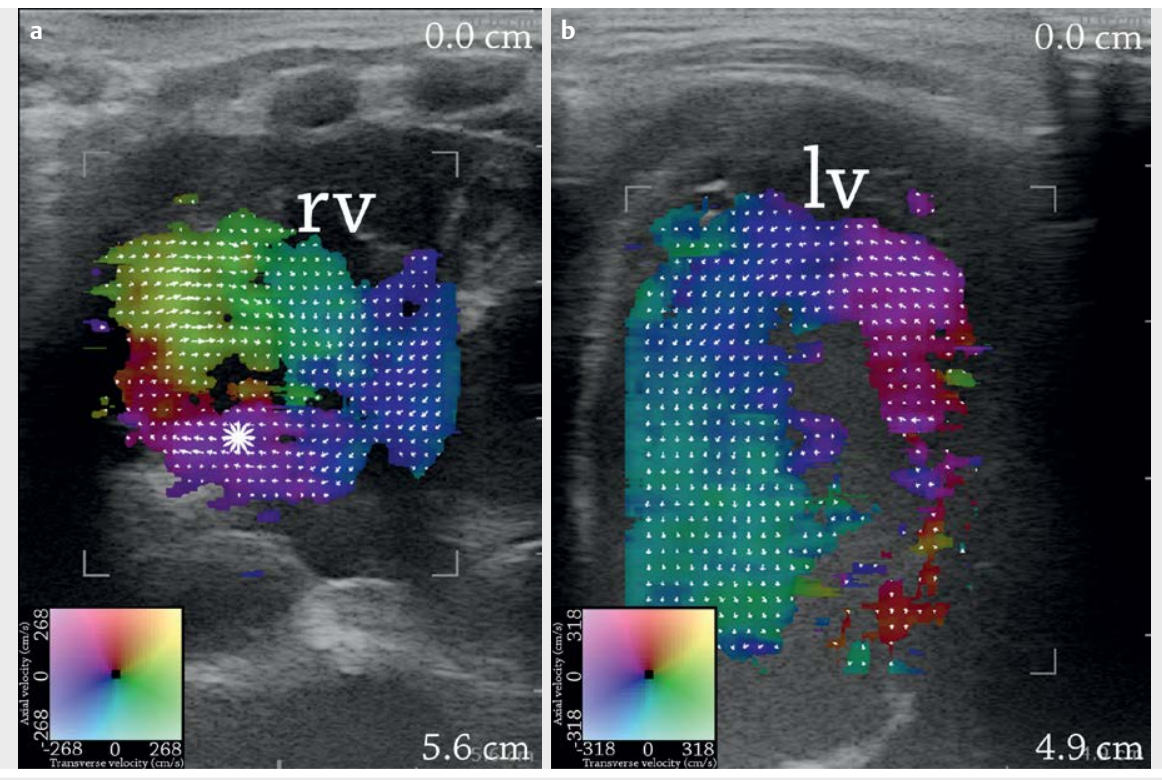

-Fig. 4 Infant with a large VSD. Left-to-right shunt through a large VSD with reversed flow in the right ventricle a, and an increased sling-like flow in the left ventricle $\mathbf{b}$. Frames are recorded in the apical view. The VSD is marked with a "star" in (a). rv= right ventricle, Iv=left ventricle.

\section{VIDEO 5}

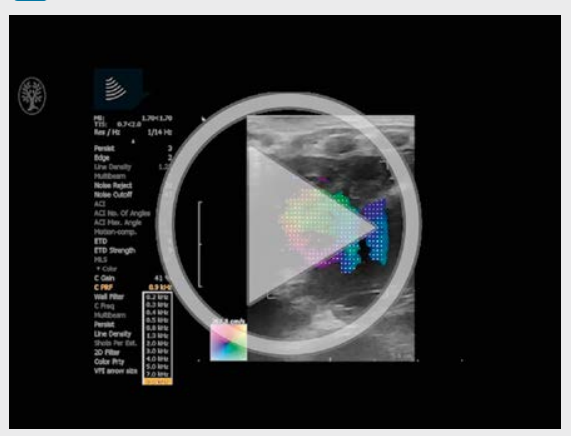

In infants with CHD, changes in flow patterns were observed. The infant with a large VSD (I) presented a significant left-to-right shunt. In the left ventricle, more pronounced flow motion was observed with a pattern as in the healthy newborns, i. e., a jet along the free wall to the apex returning along the septum to the LVOT. The shunt in the VSD was visible and created reversal of the flow in the right ventricle, i. e., the jet from the VSD combined with the flow from the right atrium followed the free wall to the apex and returned along the septum to the RVOT ( $/$ Fig. $4 \mathbf{a}$, b, and $>$ Video. 5).

For the infant with ToF (II), a right-to-left shunt was present through the VSD. The flow in the left ventricle was unchanged, but like the infant with a large VSD (I), the flow was reversed in the right ventricle with a jet along the free wall to the apex, returning along 

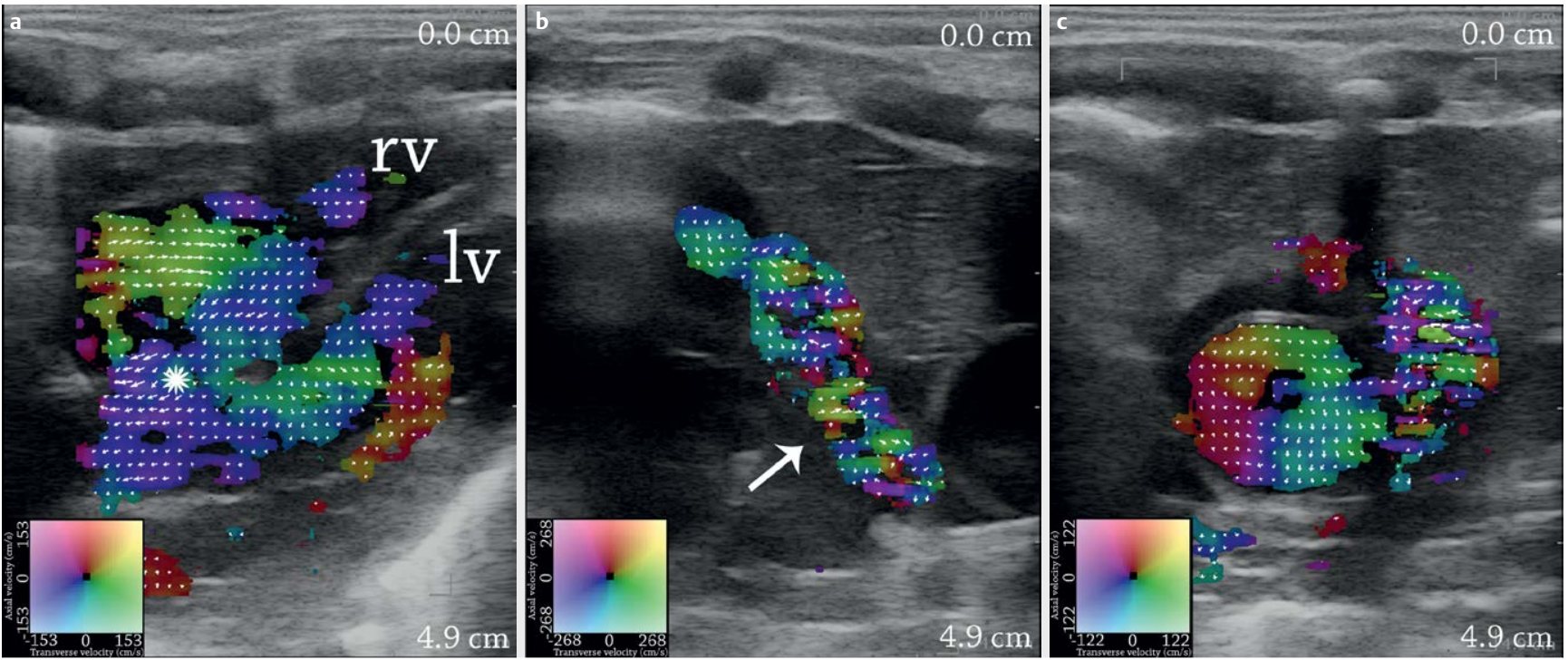

- Fig. 5 Infant with ToF. a shows the right-to-left shunt through the VSD with reversed flow circulation in the right ventricle, and b shows the complex flow pattern in the pulmonary trunk due to pulmonary constriction. $\mathbf{c}$ shows increased clockwise rotation in the ascending aorta.

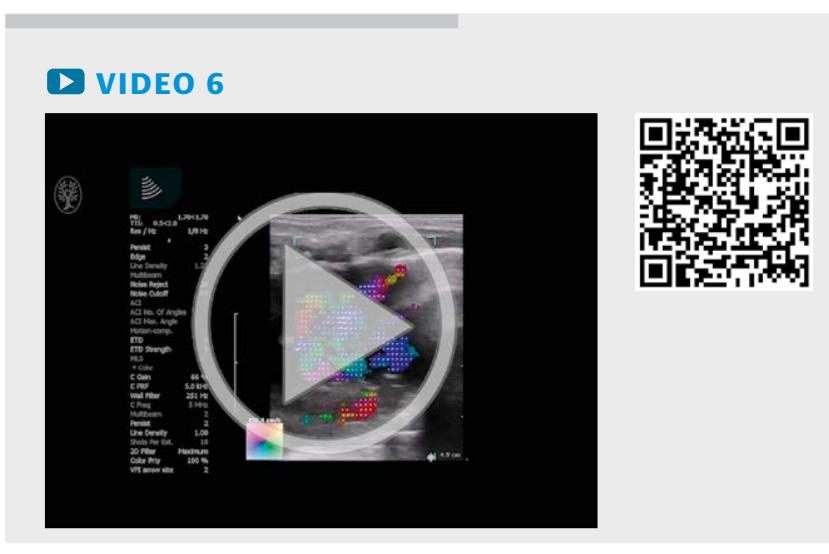

the septum to the VSD and RVOT ( Fig. 5a and $\triangleright$ Video. 6). In the RVOT, the pulmonary stenosis was seen as an increased flow complexity, when compared to the flow in RVOT of healthy newborns. The increased flow complexity was perceived as a multidirectional vector flow with color blend and arrows pointing in different directions ( $>$ Fig. 5b). In the enlarged ascending aorta, an increased clockwise secondary flow was observed, probably due to the increased flow in the aorta delivered from both the left and the right ventricle ( $\triangleright$ Fig. $\mathbf{5 c}$ ).

The infant with coarctation of the aorta (III) likewise showed increased flow complexity in the descending aorta due to the aortic narrowing ( $\triangleright$ Fig. 6a and $\triangleright$ Video. 7). The flow in the left ventricle was unchanged in terms of flow motion, and laminar flow was observed in the LVOT. In the short-axis view, Dean-like flow with opposing vortices was seen in the ascending aorta, while a clockwise rotation was seen in the left ventricle. After surgical repair, the VFI exam was repeated, and an increased aortic diameter of the descending aorta was observed with a more laminar flow. However, compared to the 6 newborns scanned over the aortic arch ( $\vee$ Fig. $2 \mathbf{c}$ ), the aortic flow after surgery still appeared with increased complex- ity ( $\triangleright$ Fig. 6b). While the flow in the left ventricle was unchanged, the secondary flow in the ascending aorta was changed to a clockwise direction after surgery.

\section{Discussion}

Previous studies using magnetic resonance imaging (MRI) have indicated vortex formation in the ventricles $[17,18]$. Whereas, the examination of healthy newborns in this study showed sling-like flow with the cardiac apex as the U-turn, and no vortical flow was observed in the four-chamber view of the ventricles. In healthy newborns, blood circulation had opposite directions in the ventricles with respect to the septum and free walls. To our knowledge, this has not been reported before. Vortical flow might be visible in other projections of the ventricles and has previously been observed in the right atrium in the adult heart using intraoperative $\mathrm{VFI}$, and in the left ventricles with a vector mapping method, where conventional Doppler data are post-processed [14, 19].

When shunts occur, the ventricular flow changes, and numerous studies with conventional ultrasound, computed tomography, and MRI have investigated these flow changes [20-22]. In this preliminary study, infants with VSDs showed reversal of the right ventricular flow despite different shunt directions in the VSDs, and in the infant with a large VSD (I), an increased sling-like flow in the left ventricle was also seen. Vector flow imaging using plane wave imaging has previously been used for shunt assessment in the pediatric heart, where shunt velocity and diameter were assessed in two patients with septal defects, although without assessment of ventricular flow changes [23].

The flow in the RVOT, LVOT and aortic arch was assessed in healthy newborns and in infants with vascular stenosis due to CHD. Compared to the normal laminar flow, stenosis of the descending aorta as well as the RVOT induced an increase in flow complexity. After surgical repair of the coarctation, improved though not normalized flow in the descending aorta was observed for the infant 

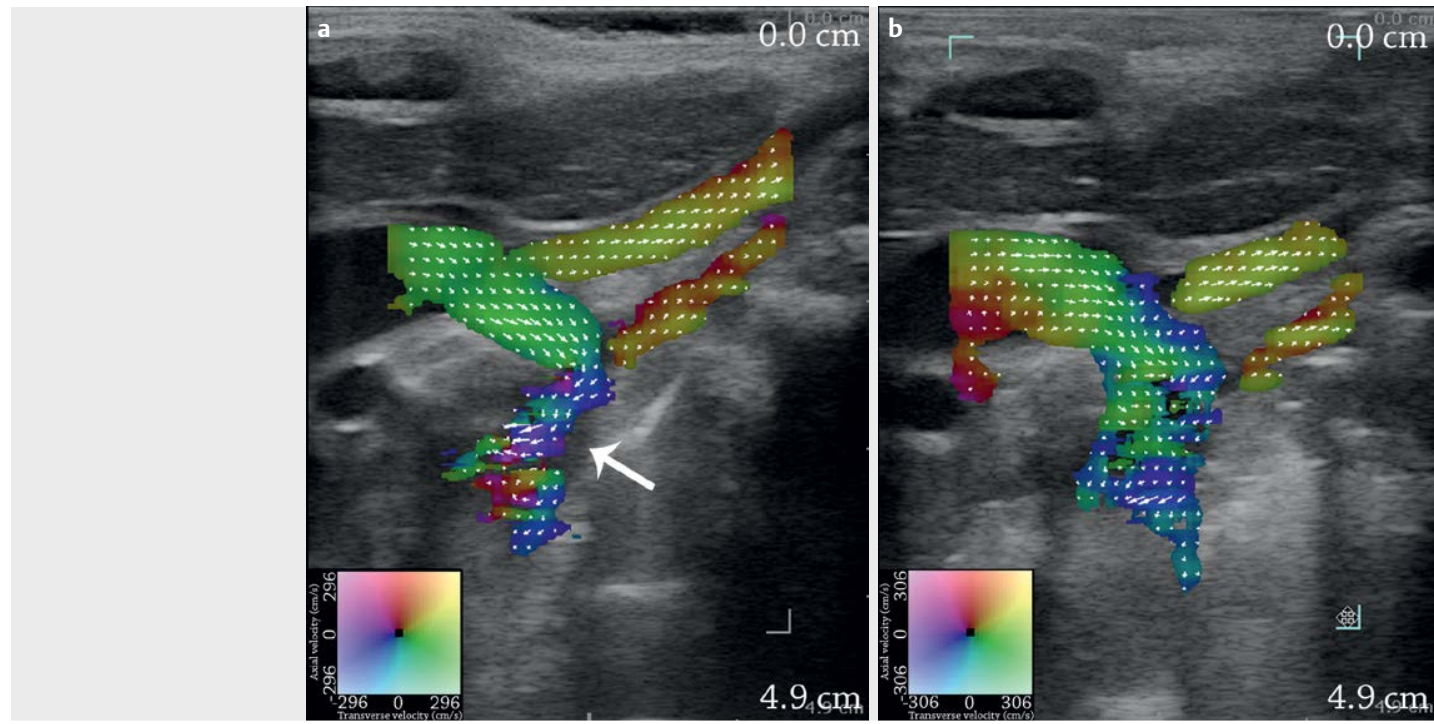

> Fig. 6 Infant with coarctation of the aorta. Complex systolic flow in the descending aorta in an infant due to aortic narrowing a. After surgical removal of the constricted vessel segment, the flow complexity is reduced $\mathbf{b}$. The frames are recorded in the suprasternal view. The coarctation is marked with an arrow in (a).
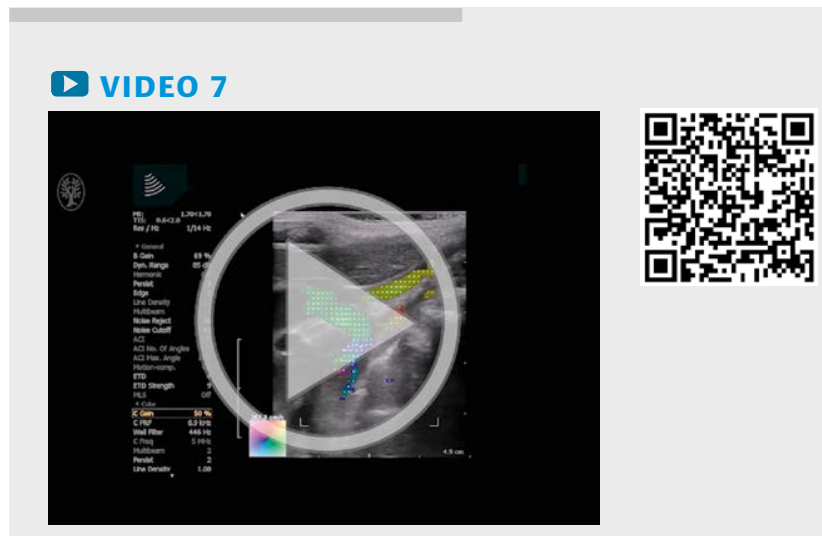

(III). Likewise, improved but not normalized flow has been observed with VFI in adult patients with aortic valve stenosis after biologic valve implantation $[9,11]$.

Conventional echocardiography assesses flow changes in constricted vessel segments by peak velocities and pressure gradients $[24,25]$. With vector velocity estimation, flow complexity can be visualized and assessed. Previous VFI studies have addressed stenosis of the aortic valve and flow changes in the ascending aorta. From vector velocity estimates, a quantitative measure for flow complexity can be derived, which previously has strongly correlated to peak systolic velocities and aortic valve stenosis $[9,11,13]$. This measure could be employed for the assessment of CHD in future studies.

The secondary rotation in the ascending aorta has been previously studied with VFI $[9-11,13,14]$. In the present study, the majority of the subjects had clockwise rotations, which is different from previous VFI studies of adults, where both clockwise and counter-clockwise rotations have been observed [9, 11]. Some of the newborns were scanned in the short-axis view over the left ventricle, and no obvious correlation to the aortic secondary flow di- rection was found. Previous VFI studies have indicated that the aortic valve is partly responsible for the secondary flow, as secondary flow changes have been observed after valve replacement $[9,11]$. In this study, one infant with coarctation of the aorta (III) showed a change in secondary flow in the ascending aorta from Dean-like to clockwise after surgery of the descending aorta. Hence, secondary flow in the ascending aorta may also be affected by surgery downstream in the descending aorta. The infant with ToF (II) had an enlarged ascending aorta with increased secondary flow compared to the healthy newborns. This is comparable to observations done in the ascending aorta in adults with aortic valve stenosis, where high antegrade velocities caused by the stenosis created faster and more pronounced secondary flow [9].

This preliminary study was achieved with a linear transducer with a limited field of view, and VFI implementation with a limited penetration depth due to the available transducer setup. However, the VFI method has been implemented on curved and phased array transducers with an increased field of view and VFI penetration, which will be employed in future studies $[26,27]$. The frame rate of the implementation is a limiting factor in cardiovascular imaging of newborns as the heart rate can easily reach 150 beats/min. This could be corrected for by using electrocardiographic gating as in MRI but is not currently integrated in the scanner [28]. The cardiac flow is highly complex, and 2D ultrasound, as used in this study, lacks the out-of-plane flow motion. To fully understand the complex flow patterns in the pediatric heart, 3D VFl is needed, and this has recently been implemented on a research scanner for flow assessment in straight vessels [29]. The current commercial VFI implementation does not provide flow quantification on the scanner, even though angle-independent flow estimation with calculation of velocity and flow direction to each pixel is obtained with VFI. So, a further developmental step for the next commercial VFI implementation should include flow quantification given directly on the scanner in order to further characterize the intracardiac or vascular flow patterns. 


\section{Conclusion}

The study indicated that VFI can be used for the assessment of cardiac flow in healthy newborns and infants with CHD. Using transthoracic VFI, common cardiac flow patterns among healthy newborns were observed along with examples of flow changes in infants with CHD.

VFI may become an integrated tool in echocardiography for pediatric cardiovascular assessment in the future. However, larger studies with improved VFI implementations are needed for further evaluation of transthoracic VFI of the pediatric heart.

\section{Conflict of Interest}

The authors declare no conflict of interest.

\section{References}

[1] van der Linde D, Konings EE, Slager MA et al. Birth prevalence of congenital heart disease worldwide: A systematic review and meta-analysis. J Am Coll Cardiol. 2011; 58: 2241-2247

[2] Bharucha T, Mertens L. Recent advances in pediatric echocardiography. Expert Rev Cardiovasc Ther 2013; 11: 31-47

[3] Deeg KH. Echocardiographic differential diagnosis of the cyanotic newborn. Ultraschall in Med 2015; 36: 104-118

[4] Berg A, Greve G. Trends in pediatric imaging: Ultrasound. Acta Radiol. 2013; 54: 1096-1105

[5] Evans DH, McDicken N, Skidmore R et al. Doppler ultrasound, Physics. Instrumentation and Clinical Applications. New York: John Wiley \& Sons; 1989

[6] Fox MD. Multiple crossed-beam ultrasound Doppler velocimetry. IEEE Trans Son Ultrason 1978; 25: 281-286

[7] Trahey GE, Allison JW, Ramm OT. Angle independent ultrasonic detection of blood flow. IEEE Trans Biomed Eng. 1987; 34: 965-967

[8] Jensen JA, Munk P. A new method for estimation of velocity vectors. IEEE Trans Ultrason Ferroelectr Freq Control 1998; 45: 837-851

[9] Hansen KL, Moller-Sorensen H, Kjaergaard J et al. Aortic Valve Stenosis Increases Helical Flow and Flow Complexity: A Study of Intra-operative Cardiac Vector Flow Imaging. Ultrasound Med Biol. 2017; 43: 1607-1617

[10] Hansen KL, Moller-Sorensen H, Kjaergaard J et al. Analysis of Systolic Backflow and Secondary Helical Blood Flow in the Ascending Aorta Using Vector Flow Imaging. Ultrasound Med Biol. 2016; 42: 899-908

[11] Hansen KL, Moller-Sorensen H, Kjaergaard J et al. Intra-operative Vector Flow Imaging Using Ultrasound of the Ascending Aorta among 40 Patients with Normal, Stenotic and Replaced Aortic Valves. Ultrasound Med Biol. 2016; 42: 2414-2422

[12] Hansen KL, Moller-Sorensen H, Kjaergaard J et al. Vector Flow Imaging Compared with Conventional Doppler Ultrasound and Thermodilution for Estimation of Blood Flow in the Ascending Aorta. Ultrason Imaging. 2017; 39: 3-18
[13] Hansen KL, Moller-Sorensen H, Pedersen MM et al. First Report on Intraoperative Vector Flow Imaging of the Heart among Patients with Healthy and Diseased Aortic Valves. Ultrasonics 2015; 56: 243-250

[14] Hansen KL, Pedersen MM, Moller-Sorensen $\mathrm{H}$ et al. Intraoperative cardiac ultrasound examination using vector flow imaging. Ultrason Imaging. 2013; 35: 318-332

[15] Jensen JA. A new estimator for vector velocity estimation. IEEE Trans Ultrason Ferroelec Freq Contr 2001; 48: 886-894

[16] Dean WR. Note on the motion of fluid in a curved pipe. Philos Mag. 1927; 4: 208-223

[17] Kilner P], Yang GZ, Wilkes AJ et al. Asymmetric redirection of flow through the heart. Nature 2000; 404: 759-761

[18] Elbaz MS, Calkoen EE, Westenberg JJ et al. Vortex flow during early and late left ventricular filling in normal subjects: Quantitative characterization using retrospectively-gated 4D flow cardiovascular magnetic resonance and three-dimensional vortex core analysis. J Cardiovasc Magn Reson. 2014; 16: 1-12

[19] Akiyama K, Maeda S, Matsuyama T et al. Vector flow mapping analysis of left ventricular energetic performance in healthy adult volunteers. BMC Cardiovasc Disord 2017, doi:10.1186/s12872-016-0444-7

[20] Gabbour M, Schnell S, Jarvis K et al. 4-D flow magnetic resonance imaging: Blood flow quantification compared to 2-D phase-contrast magnetic resonance imaging and Doppler echocardiography. Pediatr Radiol. 2015; 45: 804-813

[21] Lawley CM, Broadhouse KM, Callaghan FM et al. 4D flow magnetic resonance imaging: Role in pediatric congenital heart disease. Asian Cardiovasc Thorac Ann 2017, doi:10.1177/0218492317694248

[22] Prakash A, Powell AJ, Geva T. Multimodality noninvasive imaging for assessment of congenital heart disease. Circ Cardiovasc Imaging 2010; 3: $112-125$

[23] Fadnes S, Nyrnes SA, Torp $\mathrm{H}$ et al. Shunt flow evaluation in congenital heart disease based on two-dimensional speckle tracking. Ultrasound Med Biol. 2014; 40: 2379-2391

[24] Dijkema E], Leiner T, Grotenhuis HB. Diagnosis, imaging and clinical management of aortic coarctation. Heart 2017, doi:10.1136/ heartjnl-2017-311173

[25] Swamy P, Bharadwaj A, Varadarajan P et al. Echocardiographic evaluation of tetralogy of Fallot. Echocardiography 2015; 32: 148-156

[26] Pihl MJ, Marcher J, Jensen JA. Phased-array vector velocity estimation using transverse oscillations. IEEE Trans Ultrason Ferroelect Freq Control 2012; 59: 2662-2675

[27] Jensen JA, Brandt AH, Nielsen MB. Convex array vector velocity imaging using transverse oscillation and its optimization. IEEE Trans Ultrason Ferroelect Freq Control 2015; 62: 2043-2053

[28] Markl M, Kilner P], Ebbers T. Comprehensive 4D velocity mapping of the heart and great vessels by cardiovascular magnetic resonance. J Cardiovasc Magn Reson. 2011, doi:10.1186/532-429X-13-7

[29] Holbek S, Ewertsen C, Bouzari H et al. Ultrasonic 3-D Vector Flow Method for Quantitative In Vivo Peak Velocity and Flow Rate Estimation. IEEE Trans Ultrason Ferroelectr Freq Control 2017; 64: 544-554 